A Survey of Existing V\&V, UQ and M\&S Data and Knowledge Bases in Support of the Nuclear Energy - Knowledge Base for Advanced Modeling and Simulation (NE-KAMS)

Hyung B. Lee

December 2011

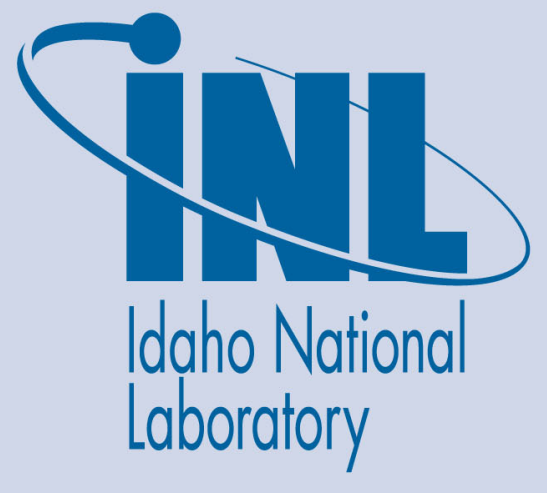

The INL is a U.S. Department of Energy National Laboratory operated by Battelle Energy Alliance 


\title{
A Survey of Existing V\&V, UQ and M\&S Data and Knowledge Bases in Support of the Nuclear Energy - Knowledge Base for Advanced Modeling and Simulation (NE-KAMS)
}

\author{
Hyung B. Lee
}

December 2011

Idaho National Laboratory
Idaho Falls, Idaho 83415

http://www.inl.gov

Prepared for the

U.S. Department of Energy

Office of Nuclear Energy

Under DOE Idaho Operations Office

Contract DE-AC07-05ID14517 


\section{EXECUTIVE SUMMARY}

The Nuclear Energy - Knowledge base for Advanced Modeling and Simulation (NE-KAMS) is being developed at the Idaho National Laboratory in conjunction with Bettis Laboratory, Sandia National Laboratories, Argonne National Laboratory, Oak Ridge National Laboratory, Utah State University and others. The objective of this consortium is to establish a comprehensive knowledge base to provide Verification and Validation (V\&V) and Uncertainty Quantification (UQ) and other resources for advanced modeling and simulation (M\&S) in nuclear reactor design and analysis. NE-KAMS will become a valuable resource for the nuclear industry, the national laboratories, the U.S. NRC and the public to help ensure the safe operation of existing and future nuclear reactors.

A survey and evaluation of the state-of-the-art of existing V\&V and M\&S databases, including the Department of Energy and commercial databases, has been performed to ensure that the NEKAMS effort will not be duplicating existing resources and capabilities and to assess the scope of the effort required to develop and implement NE-KAMS. The survey and evaluation have indeed highlighted the unique set of value-added functionality and services that NE-KAMS will provide to its users. Additionally, the survey has helped develop a better understanding of the architecture and functionality of these data and knowledge bases that can be used to leverage the development of NE-KAMS.

The survey showed that the majority of the existing data and knowledge bases are $\mathrm{V} \& \mathrm{~V}$ and M\&S databases. The V\&V databases are mostly for computational fluid dynamics and exist mostly outside of the nuclear domain. These include the European Research Community On Flow, Turbulence And Combustion (ERCOFTAC), QNET-CFD, and NPARC Alliance databases. Evaluation of these databases showed that they are essentially repositories for validation data and $\mathrm{V} \& \mathrm{~V}$ assessment results. In general, all that these databases do is maintain the validation data, which have been collected or submitted to the database over time. The quality and amount of the $\mathrm{V} \& \mathrm{~V}$ data as well as the attendant documentation vary significantly, ranging from good quality data that are well documented to poor quality data with little or no documentation. There are no standards, requirements or best practices guidance for $\mathrm{V} \& \mathrm{~V}$ and UQ associated with these databases. There is also no explicit process of quality assurance, such as procedures for assessing and classifying the quality of $\mathrm{V} \& \mathrm{~V}$ benchmark data or a review and approval procedure for entries into the database, associated with these databases.

M\&S databases, on the other hand, exist for many disciplines, including the nuclear domain. However, most M\&S databases are very specific in nature, dealing only with a specific type of data and functionality. For instance, the Gen IV Materials Handbook database at the Oak Ridge National Laboratory only deals with nuclear material properties whereas the ENDF/B database from the Cross Section Evaluation Working Group (CSEWG) only deals with nuclear cross section data. The climate modeling database of the Earth Systems Grid (ESG) at the Lawrence Livermore National Laboratories only supports climate modeling while the Environmental Management groundwater and soil databases at the Pacific Northwest National Laboratory only supports M\&S for geosciences. Most of the M\&S databases surveyed also provide little or no functionality or services related to V\&V and UQ for related modeling and simulations. 
Unlike the V\&V and M\&S databases surveyed, NE-KAMS will be a multi-purpose, web-enabled knowledge base that will provide multiple resources for V\&V, UQ and computational methods development. Specifically, NE-KAMS will:

- Establish accepted standards, requirements and best practices for V\&V and UQ of computational models and simulations,

- Establish accepted standards and procedures for assessing the quality of $\mathrm{V} \& \mathrm{~V}$ benchmark data,

- Provide readily accessible databases of quality-assessed V\&V and UQ benchmark data that can be used in V\&V and UQ assessments,

- Provide a searchable knowledge base of information, documents and data related to $\mathrm{V} \& \mathrm{~V}$ and UQ, and

- Provide web-enabled applications, tools and utilities for V\&V, UQ and M\&S activities, data assessment and processing, and information and data searches.

It is envisioned that NE-KAMS will serve as a V\&V and UQ knowledge base hub that will provide readily accessible services and provide access to a federated system of data and knowledge bases. The primary elements of NE-KAMS with the attendant hardware, software and infrastructure will be implemented in stages.

Based on the results of the survey as well as subsequent meetings with cognizant technical personnel at the national laboratories, a coherent strategy for linking NE-KAMS to other DOE databases has been developed. In addition, resources, tools and expertise within the national laboratories that can be readily utilized in the implementation of NE-KAMS have been identified. In fact, database software and expertise from three specific national laboratories have been identified for use in the NE-KAMS effort. These are:

- Gen IV Materials Handbook Database System from the Oak Ridge National Laboratory.

- Generalized Environment for Modeling Systems (GEMS) from the Idaho National Laboratory.

- VELO: A Collaborative Knowledge Management Framework for Simulation and Modeling from the Pacific Northwest National Laboratory.

The NE-KAMS effort will take advantage of two technology demonstration programs in 2012 to accelerate its development. These programs are the Nuclear Energy Advanced Modeling and Simulation (NEAMS) Pathways Use Cases and Consortium for Advanced Simulation of Light Water Reactors (CASL) Validation Data Management System. It is judged that these demonstrations will accelerate the development of NE-KAMS by providing valuable hands-on experience in developing and implementing relational databases and knowledge management systems for $\mathrm{V} \& \mathrm{~V}$, UQ and M\&S workflow capture and documentation. It is expected that the Gen IV Material Handbook database framework as well as the GEMS and VELO systems will be utilized extensively in both the NEAMS Pathways and CASL demonstrations. 


\section{Table of Contents}

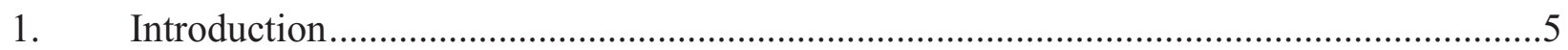

2. A Survey of Existing V\&V, UQ and M\&S Data and Knowledge Bases ........................5

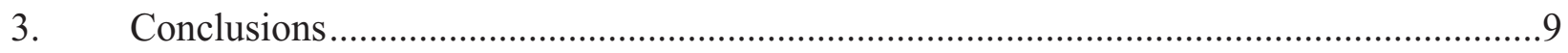




\section{Introduction}

The Nuclear Energy - Knowledge base for Advanced Modeling and Simulation (NE-KAMS) is being developed at the Idaho National Laboratory (INL) in conjunction with Bettis Laboratory, Sandia National Laboratories, Argonne National Laboratory, Oak Ridge Laboratory, Utah State University and others. The objective of this consortium is to establish a comprehensive and webaccessible knowledge base to provide Verification and Validation (V\&V) and Uncertainty Quantification (UQ) resources and support for the use of advanced modeling and simulation $(\mathrm{M} \& S)$ in nuclear reactor design, analysis and licensing. The knowledge base will serve as an important resource for technical exchange and collaboration that will enable credible computational models and simulations for application to nuclear power. It is envisioned that NE-KAMS will become a valuable resource for the nuclear industry, academia, the national laboratories, the U.S. Nuclear Regulatory Commission (NRC), and the public, and will help ensure the safe, economical and reliable operation of existing and future nuclear reactors. This document presents the results and relevant findings from the survey and evaluation of existing $\mathrm{V} \& \mathrm{~V}$, UQ and M\&S data and knowledge bases that were performed in support of the NE-KAMS effort.

\section{A Survey of Existing V\&V, UQ and M\&S Data and Knowledge Bases}

As part of the NE-KAMS effort, a state-of-the-art survey and evaluation of existing V\&V, UQ, and M\&S data and knowledge bases, including the Department of Energy (DOE), academic, and commercial data and knowledge bases, were performed to assess the scope and breadth of the effort required to develop and implement NE-KAMS, and to ensure that NE-KAMS does not duplicate resources that already exist in other data and knowledge bases. Shown below is a listing of some of the V\&V, UQ and M\&S data and knowledge bases that were surveyed and evaluated:

- ENDF/B database from the Cross Section Evaluation Working Group (CSEWG)

- International Criticality Safety Benchmark Project (ICSBEP) database

- International Reactor Physics Experiment Evaluation Project (IRPhEP) database

- European Research Community On Flow, Turbulence And Combustion (ERCOFTAC) database

- QNET-CFD knowledge base

- NPARC Alliance database

- National Agency for Finite Element Methods and Standards (NAFEMS) benchmark database

- Nuclear Energy Agency (NEA), Committee on the Safety of Nuclear Installations (CSNI) International Standards Problem (ISP) database

- NASA Aero-acoustic V\&V database

- Thermal Properties database from thermalhub.org (NSF-funded)

- Predictive Engineering and Computational Sciences (PECOS) knowledge base at the University of Texas at Austin

- Material Data Environment (MDE) database at Cornell University

- DOE Office of Scientific and Technical Information (OSTI) knowledge base

- DOE Gen IV Materials Handbook database at the Oak Ridge National Laboratory (ORNL) 
- DOE Environmental Management groundwater and soil databases at the Pacific Northwest National Laboratory (PNNL)

- DOE Earth Systems Grid (ESG) climate modeling database at the Lawrence Livermore National Laboratory (LLNL)

- DOE NGNP Data, Modeling and Analysis System (NDMAS) database at INL

The survey and evaluation were also needed to develop an understanding of the architecture and functionality of these data and knowledge bases, i.e., how the data and information are collected/acquired, reviewed, processed, formatted, stored, accessed and utilized, in order to not only leverage existing data and knowledge base efforts in the NE-KAMS development and implementation, but also to articulate the true value of NE-KAMS by highlighting the unique set of value-added functionality and services that the NE-KAMS will provide to its users.

The results of the survey show that the majority of the existing data and knowledge bases are either $\mathrm{V} \& \mathrm{~V}$ or $\mathrm{M} \& \mathrm{~S}$ databases. The $\mathrm{V} \& \mathrm{~V}$ databases are, in turn, mostly computational fluid dynamics (CFD) V\&V and assessment databases and exist mostly outside of the nuclear domain, such as the ERCOFTAC, QNET-CFD, and NPARC Alliance databases. This is not surprising since it is accurate to say that computationalists (code users and code developers) and experimentalists in the field of fluid dynamics have been the pioneers in the development of terminology, methodology, and procedures for $\mathrm{V} \& \mathrm{~V}$. M\&S databases, on the other hand, exist for many disciplines, including the nuclear domain. However, most M\&S databases are very specific in nature, dealing only with a specific set of data and functionality. For instance, the Gen IV Materials Handbook database at the ORNL only deals with nuclear material properties data and the ENDF/B database from the CSEWG only deals with nuclear cross section data.

The evaluation of the $V \& V$ and assessment databases surveyed showed that these databases are essentially repositories for $\mathrm{V} \& \mathrm{~V}$ cases, $\mathrm{V} \& \mathrm{~V}$ assessment results, and related documents. In general, all that these databases do is maintain $\mathrm{V} \& \mathrm{~V}$ cases, mostly validation cases, which have been collected or submitted to the database over time. The quality and amount of the V\&V case data as well as the attendant documentation vary significantly, ranging from good quality data that is well documented to poor quality data with little or no documentation. The users interface with the database via a website and search manually through a listing of links, directories or folders to find the $V \& V$ benchmark data and related documentation to download for use offline. There are no standards, requirements or best practices guidance for V\&V and UQ associated with these databases. There is also no explicit process of quality assurance, such as procedures for assessing and classifying the quality of $V \& V$ benchmark data or a review and approval procedure for entries into the database, associated with these databases. In fact, it can be stated that there is little or no organizational control or protocol on the operations of these databases. Once one gains access to these databases, how one uses these databases is, by and large, up to the user. Other than some brief web page documentation or an overview tutorial on $\mathrm{V} \& \mathrm{~V}$, there is no technical support services associated with these databases. It is noted that over time, most of these CFD V\&V and assessment databases have either become defunct or fallen out of date due to lack of use and interest. For most of these databases, the content of the database has not changed and there has been little or no updating of the V\&V cases in the database. It is also noted that the terms "V\&V cases" are used to describe the V\&V data in the existing databases instead of "V\&V benchmarks." This is deliberate because most of the V\&V data in these databases are not of required quality and resolution and do not have the required level of detailed 
documentation such that they can be considered to be at the level of engineering reference standard, i.e., strong sense benchmarks.

The evaluation of the M\&S databases surveyed show that these databases serve a very specific purpose and support only a specific set of $M \& S$ applications. Unlike the V\&V databases, which are, by and large, limited to CFD V\&V and assessment databases, M\&S databases exist for many disciplines, including application specific databases, e.g., materials modeling database. However, the scope of M\&S databases is very specific and limited to the specific set of M\&S it supports. For instance, the DOE climate modeling database of the ESG at the LLNL only supports climate modeling and the DOE Environmental Management groundwater and soil databases at the PNNL only supports M\&S for geosciences, e.g., groundwater flow and seismic analysis. Also, unlike most V\&V databases, which serve just as repositories for $\mathrm{V} \& \mathrm{~V}$ cases, most M\&S databases provide a significant amount of technical support services, e.g., tools and utilities for analytics and statistics, and employ state-of-the-art data and information management systems. These databases also employ operational protocol with quality assurance processes, such as procedures for assessing and classifying the quality of data or a review and approval procedure for entries into the database. Thus, although M\&S databases are generally limited in scope, they provide a significant value-added capability and support services to its users. Unfortunately, most of the M\&S databases surveyed provide little or no functionality or services related to V\&V and UQ of the M\&S that the database supports.

Unlike most V\&V and M\&S databases which either serve as repositories for a select set of data or provide support services for only a specific set of data and related M\&S applications, NEKAMS will be a general-purpose, web-enabled knowledge base that will provide value-added V\&V, UQ and computational methods development technical support for advanced M\&S. NEKAMS, when implemented, will provide significant new resources and help by:

- Establishing accepted standards, requirements and best practices for V\&V and UQ of computational models and simulations,

- Establishing accepted standards and procedures for assessing the quality of V\&V benchmark data,

- Providing readily accessible databases of quality assessed V\&V and UQ benchmark data that can be used in V\&V and UQ assessments and computational methods development,

- Providing a searchable knowledge base of information, documents and data on V\&V and UQ, and

- Providing web-enabled applications, tools and utilities for V\&V, UQ and M\&S activities, data assessment and processing, and information and data searches.

In addition, NE-KAMS will provide nuclear reactor design, analysis and licensing related data, information and utilities not found in any other data or knowledge bases:

- Focused and targeted V\&V and UQ case studies of nuclear reactor systems, components and processes to enable reduction in uncertainty and improvement in applicability of computational models and simulations used in the nuclear industry;

- Higher level data classification and categorization for nuclear reactor design and safety analysis, employing validation hierarchy (validation pyramids) and Phenomena 
Identification and Ranking Tables (PIRT) charts for applicable nuclear reactor designs and systems.

It is envisioned that NE-KAMS, when implemented, will serve as a V\&V and UQ knowledge base hub that will provide secure and readily accessible web-enabled services for accessing and utilizing a federated system of V\&V, UQ and M\&S data and knowledge bases to its users in industry, government and academia. The primary elements of NE-KAMS along with the attendant hardware, software and facility infrastructure will be developed, implemented and deployed in stages over time. In particular, a dedicated suite of NE-KAMS data and knowledge bases, e.g., CFD V\&V benchmark databases, and links and connections to other data and knowledge bases, especially those of the DOE, will need to be developed, implemented and deployed in stages over time to form the federated system of $V \& V$, UQ and M\&S data and knowledge bases that will be supported by NE-KAMS. The database survey discussed above will help in defining the scope and breadth of the NE-KAMS effort. Also, understanding the architecture and functionality of existing data and knowledge bases, i.e., how the data and information are collected/acquired, reviewed, processed, formatted, stored, accessed and utilized, will help in implementing NE-KAMS.

Based on the results of the survey and related assessments as well as subsequent meetings with cognizant technical personnel at the DOE national laboratories, a coherent strategy for linking and connecting NE-KAMS to other DOE data and knowledge bases was developed. The strategy calls for two levels of connectivity: tight integration and loose connectivity. Tight integration will be used to connect NE-KAMS to other data and knowledge bases that will be integral parts of the federated system of data and knowledge bases that NE-KAMS will support. In particular, a local, NE-KAMS V\&V and UQ database will be deployed as a part of the data or knowledge base that requires tight integration. For instance, it is envisioned that the Gen IV Materials Handbook database at the ORNL will be tightly integrated to the NE-KAMS V\&V and UQ knowledge base hub and, as such, it will host a local, NE-KAMS V\&V and UQ database which will handle all the communication and interaction between the NE-KAMS V\&V and UQ knowledge base hub and the Gen IV Materials Handbook database. Similarly, the loose connectivity approach will be used to connect the NE-KAMS V\&V and UQ knowledge base hub to those data and knowledge bases where connectivity is only required to access documents and information. For instance, it envisioned that the OSTI knowledge base will be loosely connected to the NE-KAMS V\&V and UQ knowledge base hub so that NE-KAMS users can access the large number of DOE documents and publications in the OSTI library and archives.

Based on the results of the survey, it has been determined that there exist resources, tools and expertise within the DOE national laboratories that can be readily leveraged and utilized in the development and implementation of NE-KAMS. To that end, three specific database and M\&S software systems and frameworks from the DOE national laboratories have been identified for use in the NE-KAMS effort:

- Gen IV Materials Handbook Database System from the ORNL. It is judged that the relational database framework developed at ORNL can be used readily to develop all the relational databases being considered for NE-KAMS. It is expected that the NE-KAMS effort will standardize on the relational database framework developed for the Gen IV Materials Handbook database. 
- Generalized Environment for Modeling Systems (GEMS) from the INL. It is judged that the GEMS system can be used readily to develop environments for capture and automation of workflows and related data integration. Because GEMS is composed of Commercial-Off-The-Shelf (COTS) systems, it can readily integrate COTS computer aided engineering (CAE) and office automation software, e.g., Microsoft SharePoint, SAS Analytics and Matlab.

- VELO: A Collaborative Knowledge Management Framework for Simulation and Modeling from the PNNL. It is judged that the VELO framework can be used to develop robust and scalable systems for workflow capture and documentation as it supports common knowledge management needs across modeling and simulation domains, including data storage, semantic markup, metadata extraction, provenance, versioning and access control.

The NE-KAMS effort will take advantage of two technology demonstration programs in 2012 to accelerate the development of NE-KAMS. The two technology demonstration programs are

- NEAMS Pathways Use Cases, and

- CASL Validation Data Management System

As part of each demonstration, the V\&V, UQ and M\&S workflows will be captured, documented and integrated with relational databases and semantically tagged distributed file systems. It is judged that these demonstrations will accelerate the development of NE-KAMS by providing valuable hands-on experience in developing and implementing relational databases and knowledge management systems for workflow capture and documentation. It is expected that the Gen IV Material Handbook database framework as well as the GEMS and VELO systems will be utilized extensively in both the NEAMS Pathways and CASL demonstrations.

\section{Conclusions}

A state-of-the-art survey and evaluation of existing $\mathrm{V} \& \mathrm{~V}, \mathrm{UQ}$, and $\mathrm{M} \& \mathrm{~S}$ data and knowledge bases have been performed to assess the scope and breadth of the effort required to develop, implement, and deploy NE-KAMS, and to ensure that the NE-KAMS effort does not duplicate resources and capabilities that already exist in other data and knowledge bases. The results of the survey and evaluation showed that NE-KAMS will provide a unique set of value-added functionality and services that cannot be found in any other existing V\&V, UQ and M\&S data or knowledge bases. In addition, through the survey and evaluation, resources, tools and expertise within the DOE national laboratories that can be readily leveraged and utilized in the development, implementation and deployment of NE-KAMS were identified. The NE-KAMS effort will utilize these DOE resources, tools and expertise in two technology demonstration programs in 2012 to accelerate the development of NE-KAMS. It is judged that these demonstrations will accelerate the development of NE-KAMS by providing valuable hands-on experience in developing and implementing relational databases and knowledge management systems for $\mathrm{V} \& \mathrm{~V}$, UQ and $\mathrm{M} \& \mathrm{~S}$ workflow capture and documentation. 